\title{
Directional enhancement of refractive index and tunable wettability of polymeric coatings due to preferential dispersion of colloidal $\mathrm{TiO}_{2}$ nanorods towards their surface
}

\author{
Francesca Pignatelli a,*, Riccardo Carzino a , Marco Salerno ${ }^{a}$, Marco Scotto ${ }^{a}$, Claudio Canale a , \\ Monica Distaso ${ }^{\mathrm{a}, 1}$, Francesco Rizzi ${ }^{\mathrm{a}}$, Gianvito Caputo ${ }^{\mathrm{b}}$, Pantaleo Davide Cozzoli ${ }^{\mathrm{b}, \mathrm{c}}$, \\ Roberto Cingolani ${ }^{\mathrm{a}, \mathrm{b}}$, Athanassia Athanassiou ${ }^{\mathrm{a}, \mathrm{b}, *}$ \\ a IIT, Italian Institute of Technology, Via Morego 30, 16163 Genova, Italy \\ ${ }^{\mathrm{b}}$ NNL, National Nanotechnology Laboratory, INFM, CNR, IIT Research Unit, Via Arnesano Km 5, 73100 Lecce, Italy \\ c Scuola Superiore ISUFI, Distretto Tecnologico, University of Salento, Via Arnesano Km 5, 73100 Lecce, Italy
}

\section{A R T I C L E I N F O}

\section{Article history:}

Received 4 March 2009

Received in revised form 18 November 2009

Accepted 26 January 2010

Available online 2 February 2010

\section{Keywords:}

Polymer matrix nanocomposites

Titanium oxide

Thin films

Optical properties

Wettability

Laser irradiation

\begin{abstract}
A B S T R A C T
We demonstrate the fabrication of nanocomposite coatings, of organic-capped colloidal $\mathrm{TiO}_{2}$ nanorods dispersed into a poly(methyl methacrylate) matrix, with rising value of refractive index from the bottom to the top layers, and UV-induced surface wettability alteration, in a reversible manner. This behaviour is attributable to preferential dispersion of the $\mathrm{TiO}_{2}$ nanoparticles towards the superficial layers of the coatings. Above a critical $\mathrm{TiO}_{2}$ loading, the nanorods at the surface form aggregates deteriorating the optical and the surface properties of the nanocomposites. The optimal conditions for nanocomposite films preparation in terms of optimized nanorods dispersion, optical clarity, and surface smoothness are determined.
\end{abstract}

(C) 2010 Elsevier B.V. All rights reserved.

\section{Introduction}

Embedding inorganic nanoparticles in a polymeric matrix can lead to unique nanocomposite systems exhibiting a combination and/or synergistic enhancement of the properties of the individual materials. This potentially allows detailed tailoring of the chemical-physical characteristics of any polymer so as to target specific applications by properly choosing and tuning the properties and quantity of the nanofillers.

In particular, large band gap metal oxide nanofillers in a polymeric coating are expected to improve the quality of ophthalmic and optical components, enhancing the transmitted light and reducing the reflections. The main characteristics that nanocomposite coatings should provide are high transparency in the visible range and high refractive index $[1,2]$. These properties combined with the controlled wettability of some nanocomposite films make them ideal coatings for many optical devices (from windows to optical components) [1,2].

\footnotetext{
* Corresponding authors. IIT, Italian Institute of Technology, Via Morego 30, 16163 Genova, Italy.

E-mail addresses: francesca.pignatelli@iit.it (F. Pignatelli), athanassia.athanassiou@unile.it (A. Athanassiou).

${ }^{1}$ Current address: Institute of Particle Technology, Friedrich-Alexander-Universität Erlangen-Nürnberg, Cauerstr. 4, D-91058 Erlangen, Germany.
}

Among different polymers the poly(methyl methacrylate) (PMMA) is ideal for optical applications such as optical fibers, lenses and protective coatings. Its widespread use is mainly due to its high transparency, contamination resistance and easy processability [3].

On the other side, titanium dioxide $\left(\mathrm{TiO}_{2}\right)$ is a well-known white pigment, used since ancient times [4,5]. Some of the properties that made $\mathrm{TiO}_{2}$ so widespread are biocompatibility, chemical stability, photodurability, mechanical robustness and cheapness. Among others, the capability of $\mathrm{TiO}_{2}$ to photocatalyze hazardous chemical wastes found in air, soil and water is possibly the most attractive property and it has been largely investigated $[5,6]$. Moreover, due to its high refractive index $\mathrm{TiO}_{2}$ is a suitable material for coatings of optical components or for waveguide implementations [5]. Another important property of $\mathrm{TiO}_{2}$ films or coatings is the capability to change their surface wettability under UV irradiation [7]. This property together with its photocatalytic activity can be properly exploited in functional coatings, producing self cleaning and antifogging surfaces.

UV irradiation of titanium dioxide, with energy larger than the band gap $E_{g}$, induces the excitation of electrons from the valence band to the conduction band. The excited charge carriers can recombine radiatively or nonradiatively. The photogenerated electron-hole pairs may also migrate to the surface of the material, get trapped and react with donor or acceptor molecules absorbed at the surface. In the latter 
case the reaction may ultimately lead to the destruction of organics [8].

The wettability conversion of a crystalline surface of $\mathrm{TiO}_{2}$ from quite hydrophobic (contact angle of $\sim 40^{\circ}-70^{\circ}$ ) to superhydrophilic (contact angle of $\sim 0^{\circ}$ ), upon UV irradiation, is initiated by the reaction of the photogenerated holes with surface lattice oxygens to form oxygen vacancies. The latter can promote the adsorption of hydroxyl groups on the surface after dissociation of atmospheric water, leading to a richly hydrohylated metastable surface of high hydrophilic character [6], with nanoscale domain structures [7,9-11]. The change in the samples' wettability upon UV irradiation is a fully reversible procedure. Indeed, after one month storage in the dark, at ambient condition, the hydroxyl groups are gradually desorbed from the surface and replaced by atmospheric oxygen so that the original state recovers $[7,9-11,15]$.

In order to enhance interfacial charge transfer reactions of bulk $\mathrm{TiO}_{2}$, the crystallite dimensions have been reduced and many different geometries have been investigated. Small dimensions result in a larger band gap and yield higher redox potentials. The higher surface-tovolume ratio results in a high density of active sites for surface reactions. On the other hand decreasing particle dimensions also enhances other radiative and nonradiative recombinations. Therefore, the photoactivity of $\mathrm{TiO}_{2}$ nanoparticles can be maximized only by properly controlling the size and the geometry of the nanoparticles, to have a larger material photoefficiency $[12,13]$.

Titanium dioxide in the anatase phase turned out to be the most photoactive and the most promising semiconductor oxide for "smart" coatings applications $[4,14,15]$. In most practical applications $\mathrm{TiO}_{2}$ is used in the form of a thin film, whereas little effort has been devoted up to now to the study of $\mathrm{TiO}_{2}$ embedded in organic matrix [16]. This latter form can be ideal for applications due to its easy and cheap processability.

In this paper we present a thin coating fabrication process based on spontaneous accumulation of nanoparticles towards the thin film surface upon spin coating of a composite solution containing a polymer and preformed colloidal oxide nanocrystals. In particular we studied the optical and the surface properties of nanocomposite films consisting of a PMMA matrix that incorporates anatase $\mathrm{TiO}_{2}$ nanorods (NRs) capped with oleic acid (OLAC), as a function of the NR concentration.

We show that the NRs are not uniformly distributed across the thickness of the film but tend to accumulate at the top of the film. At low $\mathrm{TiO}_{2}$ loading this results in an optically clear and uniform coating of the polymer constituted by an upper thin layer of individual $\mathrm{TiO}_{2}$ NRs dispersed in the polymeric matrix. As the saturation of the surface layer is reached, further increase in NR concentration leads to surface segregation of the $\mathrm{TiO}_{2}$ NRs with only a part of the nanoparticles distributed underneath the top layer.

We demonstrate that the nanocomposite coatings show gradually increasing refractive index from the bottom to the superficial layers, a property desired for the preparation of multilayer waveguides [17]. Furthermore the nanocomposite coatings undergo hydrophobic to hydrophilic transformation upon UV irradiation, while preserving optical transparency and, at low NR concentrations, surface smoothness.

\section{Experimental details}

All chemicals were of the highest purity available and used as received. All syntheses were carried out using the standard Schlenk line set-up. Organic-capped anatase NRs with an average diameter of $\sim 3-4 \mathrm{~nm}$ and a mean length of $\sim 25-30 \mathrm{~nm}$ were obtained by lowtemperature trimethylamino-N-oxide (TMAO)-catalyzed hydrolysis of titanium tetraisopropoxide $\mathrm{Ti}\left(\mathrm{O}^{\mathrm{i}} \mathrm{Pr}\right)_{4}$ (TTIP) [18]. In a typical synthesis, $15 \mathrm{mmol}$ of TTIP was dissolved in $70 \mathrm{~g}$ of degassed oleic acid (OLAC, Aldrich 60\%) and the resulting solution was then reacted with $5 \mathrm{ml}$ of an aqueous $2 \mathrm{M}$ TMAO solution at $100{ }^{\circ} \mathrm{C}$ for $72 \mathrm{~h}$.
The $\mathrm{TiO}_{2}$ NRs were separated from their growing mixture upon 2propanol addition and were subsequently subjected to repeated cycles of re-dissolution in toluene and precipitation with acetone to wash out surfactant residuals. Finally, optically clear $\mathrm{TiO}_{2}$ stock solutions in toluene were prepared as mother solution for deposition.

For a more complete characterization of the NRs, the amount of OLAC capping molecules was determined by a thermo-gravimetric (TG) analysis on a solid sample of $\mathrm{TiO}_{2}$ NRs isolated by precipitation with ethanol. The amount of OLAC capping molecules estimated by the TG analysis is approximately $20 \mathrm{wt}$.\%.

The $\mathrm{TiO}_{2}$ concentration of the mother solution was estimated by allowing an aliquot of $200 \mu \mathrm{l}$ to evaporate. The solid residue $\left(\mathrm{TiO}_{2}+\right.$ OLAC) was $2.8 \mathrm{mg}$, giving a concentration of $14 \mathrm{mg} / \mathrm{ml}$ for $\mathrm{TiO}_{2} \mathrm{NR}$ mother solution. This value was used for calculating the suitable volume to be used to prepare each solution at different wt.\% concentration.

In order to use a volume of toluene as small as possible, a very high concentrated solution of PMMA in toluene was prepared $(75 \mathrm{mg} / \mathrm{ml})$.

All the PMMA (Mw 120,000) based solutions with $\mathrm{TiO}_{2}$ concentrations ranging between $5 \mathrm{wt} . \%$ and $77 \mathrm{wt} . \%$ have been prepared mixing appropriate amounts of NRs and polymer mother solution aliquots. In fact, the total volume of each solution was always $1.0 \mathrm{ml}$, adding or removing by evaporation the suitable amount of toluene, with a constant polymer content equal to $16.7 \mathrm{ml}$. In this way the viscosity of the solutions allows us to get a spin-coated film thickness around $100 \mathrm{~nm}$ for each solution.

Nanocomposite thin films were prepared by spin coating (Sawatec SM-180-BT) at $1000 \mathrm{rpm}$ for $1 \mathrm{~min}$ the $\mathrm{TiO}_{2}$ NR-PMMA solutions onto properly cleaned glass or quartz (for spectrophotometer measurements) substrates. The films were dried in oven for $1 \mathrm{~h}$ at $\sim 100{ }^{\circ} \mathrm{C}$ and subsequently kept under vacuum for at least one day.

Pure NR films were prepared by Langmuir-Blodgett technique using a KSV minitrough 2 dipper (Helsinki, Finland). Monomolecular films ( $240 \mu \mathrm{l}$ of a $2.27 \mathrm{mM} \mathrm{TiO}_{2}$ NRs in toluene solution) spread on aqueous subphase, filtered water with resistivity of $18 \mathrm{M} \Omega / \mathrm{cm}$ (Milli $\mathrm{Q}$ Millipore, Billerica, MA), were transferred by vertical dipping on hydrophilic quartz substrate (surface pressure of $25 \mathrm{mN} / \mathrm{m}^{2}$ and dipping rate $2 \mathrm{~mm} / \mathrm{min}$ ). The deposition ratio was set to 0.8 for odd layers and 0.6 for even layers. The procedure was repeated five times as to deposit five layers.

For the irradiation experiments the samples were irradiated by the third harmonic wavelength $(355 \mathrm{~nm})$ of a Nd:YAG laser, with pulse duration of $10 \mathrm{~ns}$, repetition rate of $10 \mathrm{~Hz}$ and energy density of $9 \mathrm{~mJ} / \mathrm{cm}^{2}$. The energy density has been measured by an energy-meter coupled with oscilloscope (LeCroy). The laser spot with around $1.8 \mathrm{~cm}$ diameter was positioned always on the center of the film.

The surface morphology of the nanocomposite thin films was investigated by means of atomic force microscopy (AFM) with a MFP 3D instrument (Asylum Research, USA). The AFM was operated in air in contact mode (CM-AFM), using silicon probes (CSG10, NT-MDT, Russia) with standard tip (aspect ratio $\sim 2.6$, nominal radius of curvature $r \sim 10 \mathrm{~nm}$ ) and relatively soft cantilever beam (nominal spring constant $k<0.1 \mathrm{~N} / \mathrm{m}$ ). While contact mode is usually more invasive than tapping mode, due to the presence of lateral shear forces at the tip-sample interface on the horizontal plane, this mode was selected as it generally provides more reliable measurements of the vertical dimension (i.e. feature height). On the other hand, we checked for the possible appearance of sample surface modification on scanning, by zooming out and imaging again a larger sample area after each AFM measurement.

Tapping mode (TM-AFM) was operated in air using single beam uncoated silicon cantilevers (OMCL-AC160TS-W, Olympus, Japan) with tip radius of curvature $<10 \mathrm{~nm}$ and cantilever resonance frequency $\sim 300 \mathrm{kHz}$.

Wettability measurements were performed by sessile drop method using a DataPhysics OCAH 200 at laboratory conditions (temperature $22{ }^{\circ} \mathrm{C}-25{ }^{\circ} \mathrm{C}$ and relative humidity 50\%-60\%). For the 
characterization droplets of $1 \mu \mathrm{l}$ volume (drop $r \sim 0.62 \mathrm{~mm}$, surface in contact around $1.2 \mathrm{~mm}^{2}$ ) deionized water were used.

For each sample four different measurements of the contact angle were taken along the middle line of the sample. For the irradiation experiments the value of the contact angle was taken before irradiation $(t=0)$ and straight after exposing the samples to a known number of UV pulses. Along the experimental period, reference samples (one for each concentration) kept in the dark were checked.

In order to record the time dependence and rate of the wettability conversion, the water contact angles (WCA) of the samples were measured at intervals of $5 \mathrm{~min}$ exposure (3000 pulses). The total irradiation time of the samples was $60 \mathrm{~min}$. To test out the dependence of the wettability changes on the $\mathrm{TiO}_{2}$ presence, a pure PMMA film was exposed to UV light under the same conditions. No changes in wettability were found for the PMMA film.

The absorption spectra were collected with an UV-vis-NIR spectrophotometer by Varian (Cary 6000i) in double beam configuration. The absorption measurements were performed on nanocomposite films coated on quartz, using a quartz substrate in the blank channel. The absorbances of a pure polymer film spin coated on quartz, of OLAC in solution and of PMMA and OLAC drop casted on quartz were also measured.

Ellipsometric measurements were collected by a Spectroscopic Ellipsometer (MM-16 Horiba Jobin Yvon, Longjumeau, France) at an angle of incidence of $70^{\circ}$ across the spectral range $430-850 \mathrm{~nm}$.

Both thickness and optical properties were extracted from the data analysis. The optical constants of the samples have been determined using the Cauchy transparent dispersion formula.

The model used is a two-layer model, taking into account the surface roughness of the film using the Effective Medium Approximation (EMA) theory, as sketched in the inset of Fig. 2(a). The refractive indices and the thickness of the two layers were allowed to vary independently, imposing the refractive indices to be larger or equal than that of PMMA ( $n=1.49$ at $600 \mathrm{~nm}$ ) and the total thickness to be close to the measured values $(t \sim 100 \mathrm{~nm})$. Measurements were repeated at different surface points for each sample to check for the homogeneity along the surface of the samples.

\section{Results and discussion}

\subsection{Optical characterization}

The absorption spectra of the PMMA- $\mathrm{TiO}_{2}$ NR thin films obtained at different $\mathrm{TiO}_{2}$ concentrations in solution, shown in Fig. 1, give evidence for clear and transparent thin films over a wide compositional range. In the investigated spectral interval $(\lambda>250 \mathrm{~nm})$ both the polymer and the OLAC capping molecules are highly transparent.

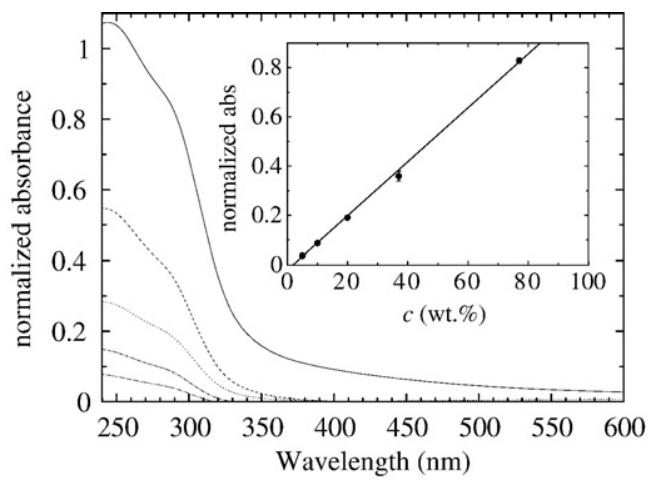

Fig. 1. Absorption spectra normalized to the thickness, at different NR concentrations, respectively $77 \mathrm{wt} . \%, 37 \mathrm{wt} . \%, 20 \mathrm{wt} . \%, 10 \mathrm{wt} . \%$ and $5 \mathrm{wt} . \%$ from the upper to the lower spectrum. In the inset the absorbance at $288 \mathrm{~nm}$ versus the NR concentration is shown.
The nanocomposite $\mathrm{PMMA}^{-\mathrm{TiO}_{2}} \mathrm{NR}$ thin films were found to be transparent in the visible range. Only the sample at $c=77 \mathrm{wt} . \%$ shows a tail extending in the visible possibly likely due to scattering from micrometer-sized $\mathrm{TiO}_{2} \mathrm{NR}$ aggregates formed within the films. The NR concentration in the starting solution, $c$, is defined as the ratio between the NR weight and the total weight of NRs and PMMA. Applying a standard peak finder routine in this range, two peaks are found at $245 \mathrm{~nm}$ and at $288 \mathrm{~nm}$. In order to exclude the polymer and the OLAC contributions we considered only the peak at $288 \mathrm{~nm}$. As shown in the inset of Fig. 1 the absorption at this wavelength is found to increase linearly with the NR concentration in the starting solutions, $c$. The thickness of the samples, measured by the profilometer and confirmed by AFM measurements, ranges between $70 \mathrm{~nm}$ and $150 \mathrm{~nm}$ increasing with the NR concentration.

The tendency of the NRs to accumulate on the top part of the nanocomposite films, see sketch in Fig. 2(b), is clear from the analysis of the ellipsometric measurements. In order to have a good fit of the measured refractive index $n$ and extinction coefficient $k$, the samples had to be modeled by a two-layer system on the glass substrate with a rough boundary region between the two layers. The rough edge was considered, using the Effective Medium Approximation (EMA) theory, by an intermediate layer composed of part of the top layer material and part of the bottom layer material. Models involving a continuous increment, either linear and parabolic, of the refractive index were also tested, resulting in a worst fit of the experimental data. The roughness of the outermost exposed surface of the film was also taken into account using the EMA theory. Such region was regarded as composed partially of air and partially of the material of the top layer. In such way we obtain two different values for the refractive index one for the top layer, $n_{t}$, and one for the bottom layer, $n_{b}$, with $n_{t}>n_{b}$,
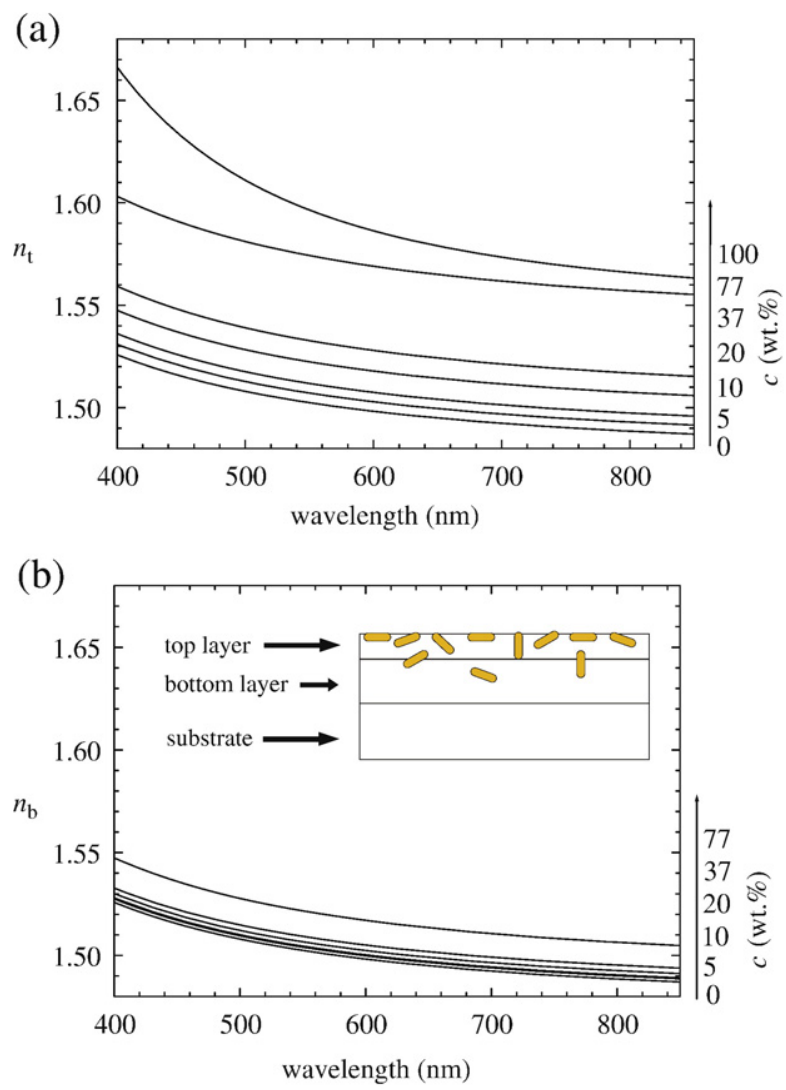

Fig. 2. Variation of the refractive index of the top and bottom layers at the different NR concentrations in the starting solutions, $c$, in the wavelength range of $400-850 \mathrm{~nm}$ : (a) Top layer refractive index; $c$ ranging, from top to bottom curve, from $100 \mathrm{wt} . \%$ to 0 wt.\%. (b) Bottom layer refractive index; $c$ from top to bottom curve ranging from 77 wt.\% to 0 wt.\%. In the inset of panel (b) a sketch of the used model is shown. 
and the respective thickness values. The refractive indices of the nanocomposites in the two layers forming the films, determined by the Cauchy dispersion formula are displayed in Fig. 2. The difference between the two layers clearly reflects the inhomogeneity in the direction perpendicular to the surface, due to the preferential accumulation of the NRs on the top of the films.

In previous works a linear dependence of the refractive index with the $\mathrm{TiO}_{2}$ concentration has been found for doped organic matrices $[19,20]$. According to our results the dependence is linear for the bottom layer and for the top layer as shown in Fig. 3. Assuming the refractive index to be independent of the film thickness, and taking into account that the refractive indices for the top and bottom layers, $n_{t}$ and $n_{b}$, linearly increase with the NR concentration on the starting solutions, $c$, and that the variation of $n$ is proportional to the concentration of NRs in each layer, i.e.: $c_{b} / \Delta n_{b}=c_{t} / \Delta n_{t}$, where $b$ stands for the bottom layer and $t$ for the top one, we derived that the ratio of the concentrations of the two layers is constant, $c_{t} / c_{b} \sim 4$. Therefore, in the studied range of $c$, the NR concentrations in the top and in the bottom layers increase proportionally as the amount on NRs in the starting solution is increased.

In Fig. 4 we report the results for the percentage thickness of the two layers, as estimated by the ellipsometer analysis. From Fig. 4 we note that for $c$ values up to $c=20 \mathrm{wt}$.\%, the thickness of the top layer is nearly constant, around $40 \mathrm{~nm}$, and of the order of the mean NR length. In this low concentration range, the NRs fill mainly a thin top layer whereas a smaller fraction of them is accommodated in the remaining nanocomposite volume underneath. Work is in progress to check whether this preferential distribution depends on the surfactant or on the polymeric matrix properties.

For higher NR concentrations, a progressive increase in the thickness of the top layer is calculated from the ellipsometric model. Possibly in this larger concentration range the NRs are forced to occupy a thicker top layer as the $40 \mathrm{~nm}$ surface coating becomes over-saturated.

The surface segregation of NRs in the top layer has direct impact on the surface morphology of the nanocomposite films, as shown by the surface topography images obtained working in contact mode AFM (CM-AFM).

For low NR concentrations, $c \leq 20 \mathrm{wt} . \%$, the films present a quite smooth surface (Fig. 5(a)). For larger NR concentration, $c=25$ wt.\%, clusters of large lateral size (several hundreds nm) get evident, Fig. 6(a). Further increase in the NR concentration leads first to an increase in the density of clusters on the surface, and finally, for concentrations $c>37 \mathrm{wt} . \%$ the clusters on the surface tend to coalesce into aggregates of larger lateral size, Fig. 6(b).

According to the AFM measurements clusters start to appear on the film surface as the concentration on the top layer overcomes a

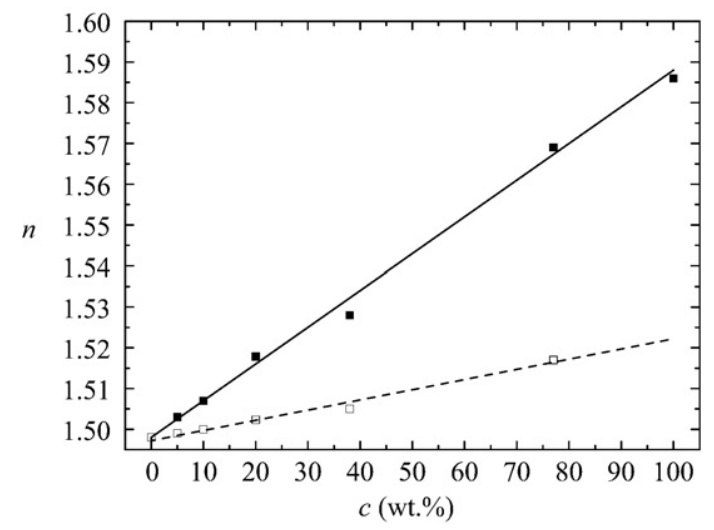

Fig. 3. Variation of the refractive index at increasing $\mathrm{TiO}_{2} \mathrm{NR}$ concentration in the starting solutions, $c$, at $600 \mathrm{~nm}$. Full and open squares refer respectively to top and bottom layers. Full and dashed lines correspond respectively to the linear fits for the top and the bottom layer data.

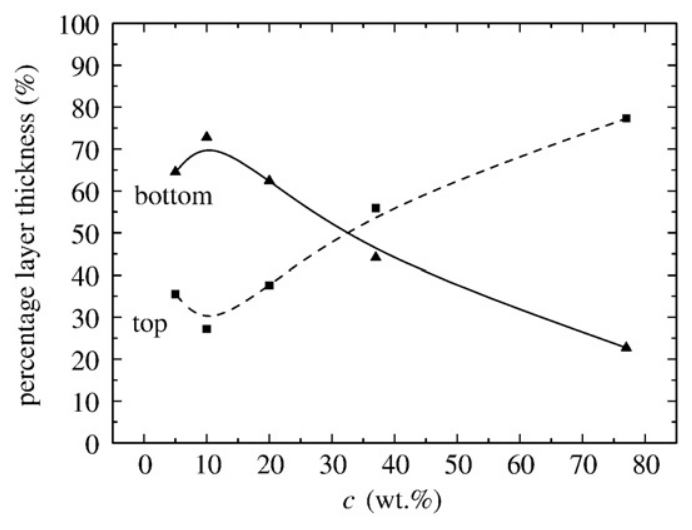

Fig. 4. Estimated thicknesses of top and bottom layers normalized to the film thickness, respectively black squares and triangles. The lines are just eye guides.

critical value of $c=20$ wt.\%. This is the same concentration at which the ellipsometric measurements indicated an increase in the top layer thickness. Therefore, we can safely assume that at that specific concentration the $40 \mathrm{~nm}$ top layer is already saturated by the NRs, and any further increase in the number of NRs leads to extensive NR aggregation on the surface. Therefore, some NRs start to organize in larger clusters.

For the lower concentrations, $c \leq 20 \mathrm{wt} . \%$, we expect individual NRs dispersed on the top surface to be visible. In order to better

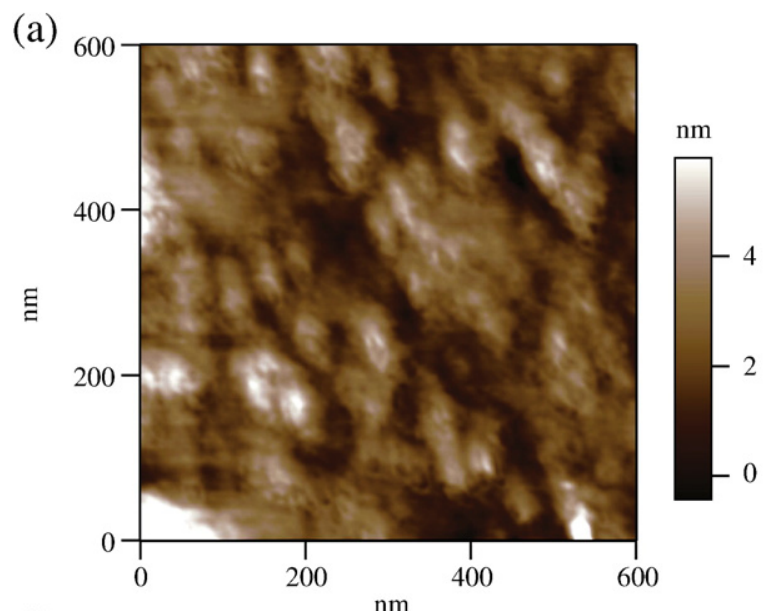

(b)

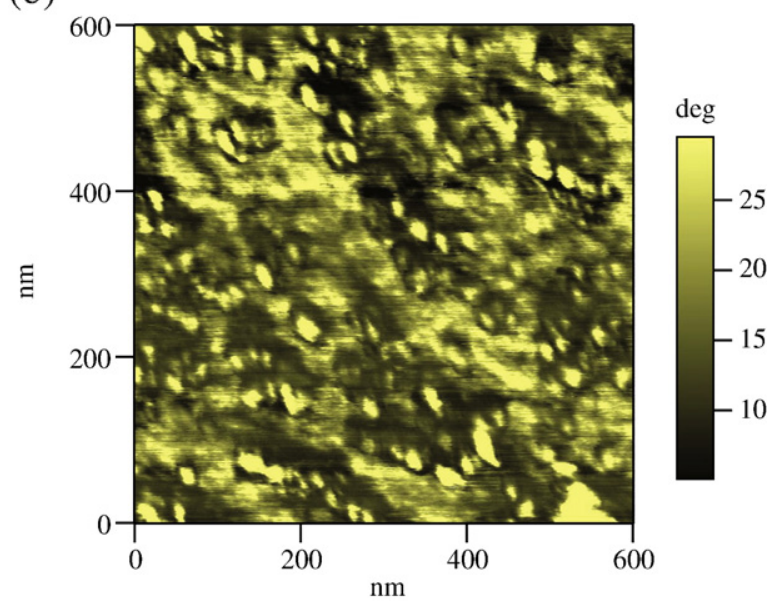

Fig. 5. (a) $600 \mathrm{~nm} \times 600 \mathrm{~nm}$ AFM topographic image of the $\mathrm{PMMA} \mathrm{TiO}_{2} \mathrm{NR}$ films at a $\mathrm{NR}$ concentration of $5 \mathrm{wt} . \%$, and (b) in the phase image of the same area, NRs and PMMA exhibit a clear contrast. 
(a)

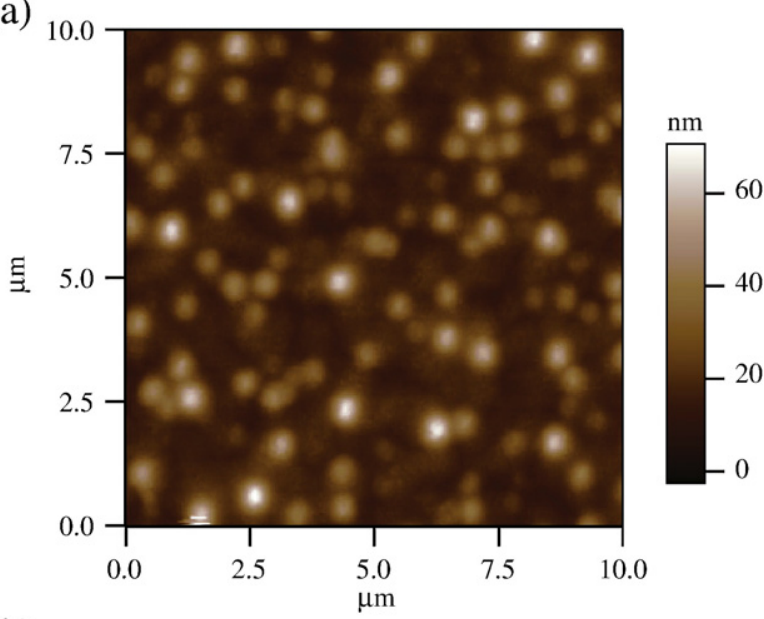

(b)

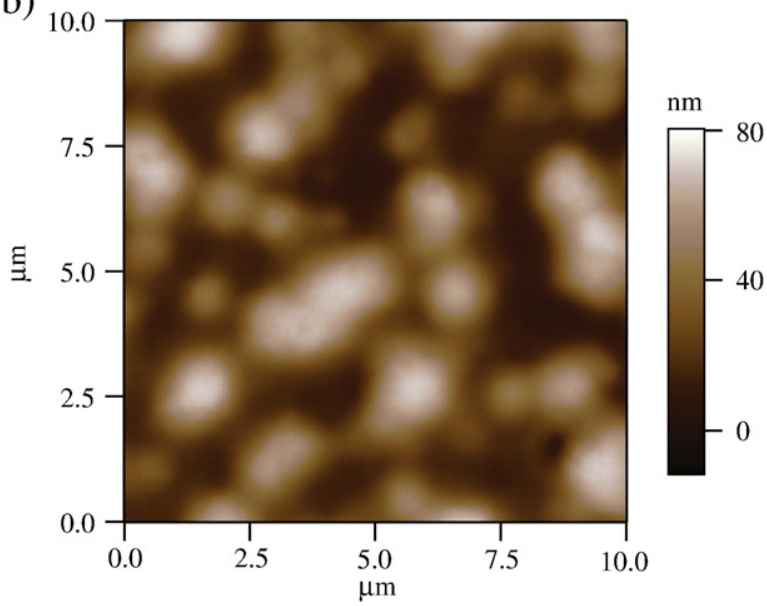

Fig. 6. $10 \mu \mathrm{m} \times 10 \mu \mathrm{m}$ AFM plane view images of the $\mathrm{PMMA} \mathrm{TiO}_{2} \mathrm{NR}$ films in the high $\mathrm{NR}$ concentration range: (a) 25 wt.\% and (b) 77 wt.\%.

differentiate between PMMA matrix and $\mathrm{TiO}_{2}$ NR phases on the top of the nanocomposite films with lower NR concentrations, tapping mode AFM (TM-AFM) has been performed. In fact, by using an oscillating probe, the simultaneous detection of the topography and the phase shift between excitation force and probe response is known to provide a qualitative mapping of the compositional domains in heterogeneous samples [21-25].

The presence of dispersed NRs on the PMMA surface could be clearly detected at low NR concentrations, see Fig. 5(b). On increasing the NR concentration, the surface coverage of dispersed NRs on the top layer also increased up to $c=20 \mathrm{wt}$.\%, that is the highest concentration for which individual NRs could be observed on the sample surface.

\subsection{Wettability and photoinduced hydrophilic conversion process}

The preferential accumulation of the $\mathrm{TiO}_{2}$ NRs towards the surface layers of the nanocomposite films affects the surface composition, and as a consequence it may also affect the surface properties of the prepared samples. Herein, we present the impact of this behaviour on the wettability of such surfaces. First the water contact angles $\left(W C A_{S}\right)$ of the PMMA/NR composite films at different NR concentrations were measured on as-prepared samples, before any irradiation $\left(W C A_{n o} U V\right)$, see Fig. 7. The WCA of a NR-only coating is also demonstrated in the figure.

At low $c$ the hydrophobicity of the different films increases with the NR concentration up to a critical concentration (37 wt.\%). At this concentration the maximum value of the contact angle of $100^{\circ}$ is reached coincident with the one of the pure NR films. Increasing

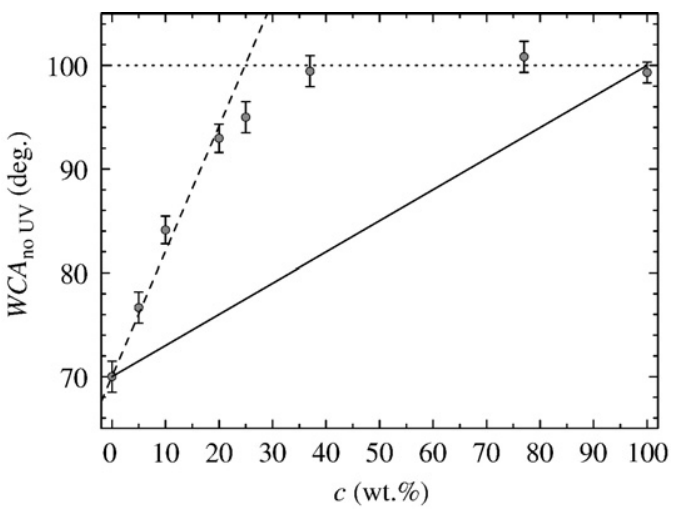

Fig. 7. Measured contact angles, $W C A_{n o} U v$, on the $\mathrm{PMMA} \mathrm{TiO}_{2}$ films, for the different NR concentrations (in solution), $c$. The dashed line corresponds to the linear fit of the experimental data, $c>25 \mathrm{wt} . \%$, and the solid line the hypothetical uninterrupted behaviour.

further the NR concentration does not change significantly the value of the contact angle, that remains around $100^{\circ}$.

The dependence of the contact angle on the NR concentration is linear up to a concentration of $20 \mathrm{wt}$.\%. The slope of this linear function is 1.2 , four times higher than the one of a hypothetical uninterrupted linear increase of the $W C A$ from the value $70^{\circ}$ to the value $100^{\circ}$, that are the values for $0 \mathrm{wt} . \%$ and $100 \mathrm{wt} . \%$ of NRs in the nanocomposite solution, respectively. Therefore, it can be assumed that similarly to the rate of the WCA changes, also the rate of NR accumulation towards the surface is elevated for NR concentrations up to $20 \mathrm{wt}$.\%.

This assumption is in agreement with the ellipsometer analysis and with the AFM measurements, that suggest an increased accumulation rate of NRs on the film surface at the low concentrations, $c \leq 20 \mathrm{wt}$.\%. At a concentration of $c=25 \mathrm{wt}$.\% the contact angle already deviates from the linear dependence, pointing out a change in the organization of the NRs in the top layer and therefore in the surface characteristics. At larger concentrations, $c>25 \mathrm{wt} . \%$, a plateau in the WCA is reached and no further changes in wettability are induced.

To explore further the surface characteristics that affect the water contact angles, we studied also the changes in the surface wettability upon UV irradiation. In Fig. 8(a) the dependence of the WCA on the irradiation time for the different NR concentrations is shown.

As previously found for coatings of pure NRs, pulsed UV irradiation induces large changes in the wettability of their surface from a hydrophobic to strongly hydrophilic state. Pulsed irradiation was found to preserve the integrity of the NR organic capping molecules inducing only conformational rearrangements of the ligand chains [26]. The pulsed nature of the excitation provides the time for electron-hole recombination (occurring in about $100 \mathrm{~ms}$ ) avoiding the production of oxidant radicals that usually drive the photocatalytic action under $\mathrm{cw}$ irradiation. The irradiation conditions were chosen so as to avoid absorption from OLAC capping molecules as well as to limit heating of the sample [26]. At the chosen irradiation wavelength $(355 \mathrm{~nm})$ both the polymer and the OLAC capping molecules do not exhibit absorption.

The doping of the polymer films with increasing number of NRs gradually alter the wetting behaviour of the polymer upon UV irradiation (no changes in wettability after UV irradiation) towards the one of the $\mathrm{TiO}_{2}$ NRs (strongly switchable wettability). At low NR concentrations the minimum contact angle reached upon UV irradiation, $W C A_{\min }$, remains quite high, just below $\sim 70^{\circ}$. In this case the exposure time needed for the process was about $60 \mathrm{~min}$. With increasing NR concentration, the $W C A_{\min }$ strongly decreases together with the irradiation exposure time needed for the conversion. The smallest $W C A_{\min }, 15^{\circ}$, measured on the NR-only coating was reached after $35 \mathrm{~min}$ exposure. Upon one month storage in the dark 
(a)

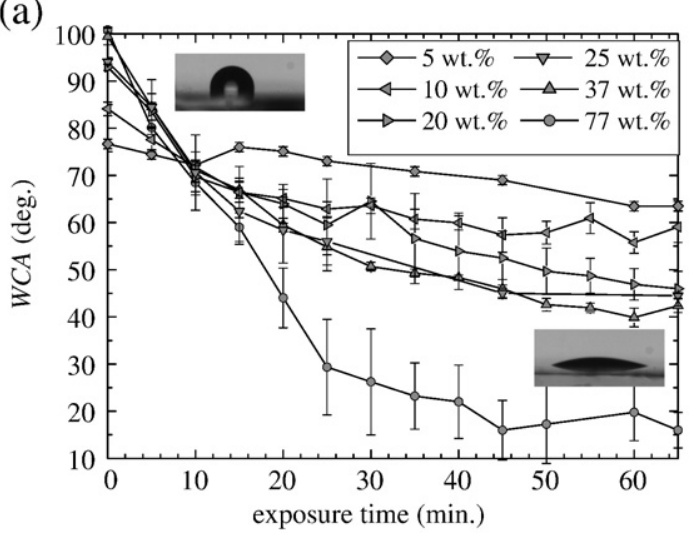

(b)

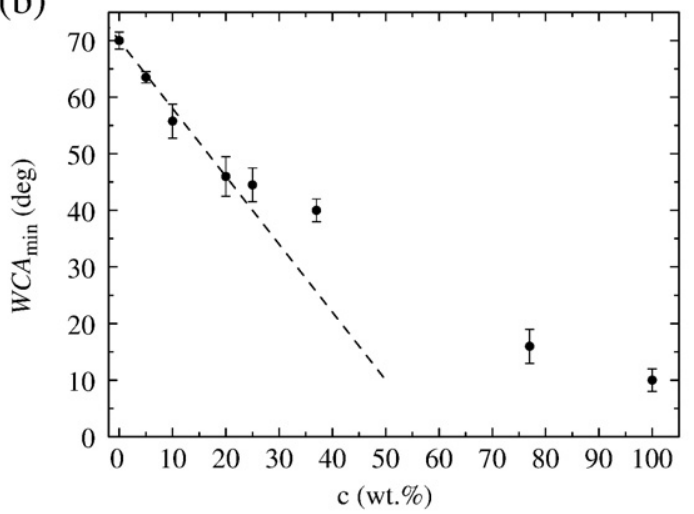

Fig. 8. (a) Water contact angle, WCA, at different irradiation steps for the different NR concentrations, $c$. (b) Dependence of the minimum water contact angle, $W C A_{\min }$, on the NR concentration in solution, $c$. The dashed line corresponds to the linear fit of the experimental data, $c<25$ wt.\%.

the samples recover their initial hydrophobic properties. On examining three cycles of UV irradiation/dark storage alternations, the phenomenon of the reversible wettability was found to be repeatable without any apparent fatigue.

The wettability changes recorded for all of the nanocomposite films are fingerprints of NRs protruding from the film surface. Indeed, if the polymer covers the NR facets emerging from the film surface, the process of hydroxyl groups absorption would be prevented, and no changes in wettability would be recorded.

In Fig. 8(b) the minimum contact angle, $W C A_{\min }$, for the different NR concentrations is reported. The minimum contact angle linearly decreases with increasing NR concentration up to $c=20 \mathrm{wt} . \%$. This linear decrease has a negative slope of -1.2 . Comparing this result with the experimental data of $W C A_{n o} U V$, it is clear that the two linear behaviours coincide in value and are just opposite in sign. This is another indication that at low concentrations, $c \leq 20 \mathrm{wt} . \%$, the NR coverage of the film increases linearly with increasing concentration.

Differently from the behaviour of the $W C A_{n o}$ uv at higher concentrations, the minimum contact angle continues to decrease with the NR concentration, with a lower rate. Combining the above results with the conclusions from the ellipsometer analysis and the AFM we can safely assume the existence of two concentration ranges at which the samples behave in a different way. In the first range, $c<25 \mathrm{wt} . \%$, the NRs linearly accumulate on the film surface, possibly obtaining a high coverage around $c=20-25$ wt.\%. At higher concentrations, $c \geq 25 \mathrm{wt} . \%$, the NRs continue to accumulate on the film surface, but in a less pronounced manner and not linearly.

Indeed, according to the ellipsometer analysis, the surface layer reaches its saturation when the top layer thickness becomes $\sim 460 \mathrm{~nm}$ at the concentration of 20 wt.\% (see Fig. 4). The film morphology changes dramatically as the NRs accumulate underneath this first NR layer, forcing at the same time the surface to organize into clusters of aggregated NRs, see Fig. 6.

According to the AFM measurements these clusters have heights of few tens of nanometers, making the surface of the nanocomposites quite rough. There is a well-known theoretical model expressed by Cassie and Baxter, which predicts the contact angles of liquids in contact with rough surfaces [27]. This model assumes that the liquid drops, in our case water drops, that come in contact with the rough surface remain on the top of the features protruding out of the surface, without being able to permeate within the roughened surface. The reason why the drops remain on the top of the rough features is that air pockets are trapped underneath the liquid. Therefore, any water drop placed on the rough surface actually sits on a composite surface made of solid and air. In this case, the water contact angle, $\theta_{C B}$, is an average between the value on $\operatorname{air}\left(180^{\circ}\right)$ and the value on the flat solid $(\theta)$ and is given by:

$\cos \theta_{C B}=f(1+\cos \theta)-1$

where $f$ is the solid fraction of the composite surface experienced by the liquid and is always smaller than unity. The Cassie-Baxter model predicts an enhancement of the WCA of the rough surface with respect to the flat one, independently from the latter. We also observe such an increase upon increasing the NR concentration in Fig. 7, which cannot be attributed to the hydrophobic character of the added $\mathrm{TiO}_{2} \mathrm{NRs}$, since the $W C A$ of anatase $\mathrm{TiO}_{2}$ is $40^{\circ}-70^{\circ}$ [4] and $\sim 75^{\circ}$ for OLAC-coated $\mathrm{TiO}_{2}$ [26]. Therefore, the wetting behaviour of our samples can be safely interpreted with the Cassie-Baxter model. Since as demonstrated in Fig. 7 the WCA reaches a plateau for NR concentrations above $37 \mathrm{wt} . \%$, we assume that in this concentration range the contact area between the water and the clusters of the protruding nanocrystals, given by $f$, remains unaltered.

After the irradiation of the samples with UV light the photogenerated holes create oxygen vacancies at the surface regions of the $\mathrm{TiO}_{2}$ NR OLAC-free. Such defect sites have the tendency to be reconstructed upon dissociative $\mathrm{H}_{2} \mathrm{O}$ adsorption, resulting in two adjacent TiO-H groups per each healed vacancy $[28,29]$. Due to its surface hydroxylation the typical WCA of clean anatase $\mathrm{TiO}_{2}$ films after UV irradiation is about $0^{\circ}$. In the case of our samples, the water drops after UV irradiation experience a sample with alternating superhydrophilic (related to the introduced $\mathrm{TiO}-\mathrm{H}$ groups) and quite hydrophobic domains (related to the OLAC-protected $\mathrm{TiO}_{2}$ areas and to the polymer covered areas). These alternating domains are expected to lead to capillary infiltration of the water drops, forcing them to spread out onto the surface of the samples. In this case the water drops are assumed to wet completely the surface of the UVirradiated films without air being trapped underneath. Therefore, the

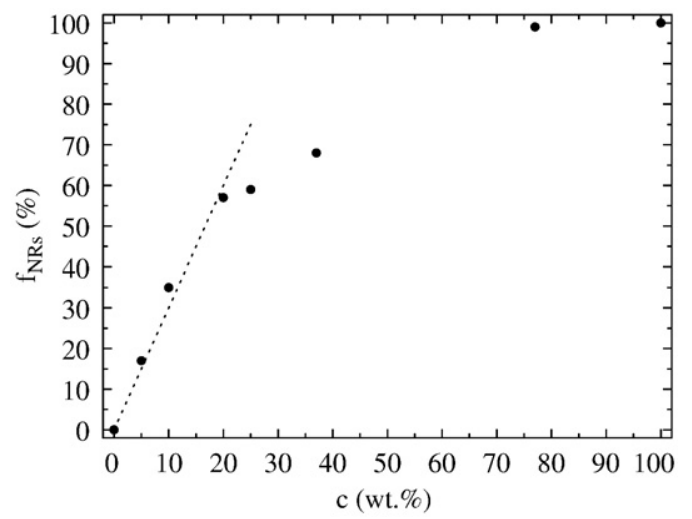

Fig. 9. Fraction of NR coverage on the surface. The dotted line is the linear fit for $c \leq 20 \mathrm{wt} . \%$. 
measured WCA values should average the WCA of the partly hydroxylated and partly OLAC-covered clusters of $\mathrm{TiO}_{2} \mathrm{NRs}$, and that of the polymer, as described by Eqs. (2) and (3).

$\cos \theta_{\text {min }}=f_{\text {NRs }} \cos \theta_{\text {NRs }}+f_{P M M A} \cos \theta_{P M M A}$

$f_{\text {NRS }}+f_{\text {PMMA }}=1$

where $\theta_{N R s}$ and $\theta_{P M M A}$ are the minimum WCA values on the pure NR layer, $15^{\circ}$, and on the pure PMMA, $70^{\circ}, f_{N R s}$ is the NR fraction, and $f_{P M M A}$ is the polymer fraction of the composite surface experienced by the water drop.

From Eqs. (2) and (3) we can calculate the fraction of the surface that is covered by NRs. This fraction is shown in Fig. 9. In full agreement with the previous assumptions presented in the paper, Fig. 9 demonstrates that the increase of the surface coverage by the NRs is linear and with an enhanced rate, for the NR concentration range in the polymer from 0 to $20 \mathrm{wt} . \%$. Above this concentration the NRs continue to accumulate on the surface but in a clearly different manner. In particular the coverage of the surface exhibits a behaviour far from the linear one, and a much slower NR accumulation. This can be attributed to the organization of the NRs in clusters, as demonstrated by the AFM.

\section{Conclusions}

We have identified a range of NR concentration in the starting nanocomposite solution which allows for the spin coating of a well dispersed nanocomposite two-layer thin film with high refractive index and with tunable hydrophobic-hydrophilic surface upon UV irradiation and dark storage cycles. The experimental analysis of nanocomposite films at different NR concentrations ranging from $c=0 \mathrm{wt} . \%$ to $100 \mathrm{wt} . \%$ gives evidence for the formation of a film consisting of two layers. At low concentrations, the top layer exhibits a smooth surface and high refractive index together with quite large and reversible surface hydrophobicity. The bottom layer nearly coincides with a pure polymer film. Clustering of particles and rough surface that is a recognized problem in these nanocomposites, happens at higher NR concentrations. We show clearly that in fact the formation of clusters is due to the exceedingly high NR concentration on the surface. This is due to the tendency of NRs to accumulate at the top layer of the film. We were able to overcome this problem by properly dosing the NR concentration according to the film thickness, so as to obtain higher refractive index together with larger hydrophobicity, without sacrificing the surface quality of the nanocomposite film.

\section{References}

[1] K.C. Krogman, T. Duffel, M.K. Sunkara, Nanotechnology 16 (2005) 338.

[2] J.L.H. Chau, C.-T. Tung, Y.-M. Lin, A.-K. Li, Mater. Lett. 62 (2008) 3416.

[3] H.A. Hornak, Polymers for Lightwave and Integrated Optics, Marcel Dekker, New York, 1992.

[4] K. Hashimoto, H. Irie, A. Fujishima, Jpn. J. Appl. Phys. 44 (2005) 8269.

[5] X. Chen, S.S. Mao, Chem. Rev. 107 (2007) 2891.

[6] O. Legrini, E. Oliveros, A.M. Braun, Chem. Rev. 93 (1993) 671.

[7] R. Wang, K. Hashimoto, A. Fujishima, Nature 388 (1997) 431.

[8] I.P. Parkin, R.G. Palgrave, J. Mater. Chem. 16 (2005) 1689.

[9] A. Mills, N. Elliott, I.P. Parkin, S.A. O'Neill, R.J. Clarck, J. Photochem. Photobiol. A Chem. 151 (2002) 171.

[10] T. Kemmitt, N.I. Al-Salim, M. Waterland, V.J. Kennedy, A. Markwitz, Curr. Appl. Phys. 4 (2004) 189.

[11] M. Miyauchi, A. Nakajima, A. Fujishima, K. Hashimoto, T. Watanabe, Chem. Mater. $12(2000) 3$.

[12] Z. Zhang C.C. Wang R. Zakaria, J.Y. Ying J. Phys. Chem. B 102 (1998) 10871.

[13] H. Kominami, S.Y. Murakami, J.I. Kato, Y. Kera, B. Ohtani, J. Phys. Chem. B 106 (2002) 10501.

[14] D. Wang, B. Yu, J. Hao, W. Liu, Mater. Lett. 62 (2008) 2036

[15] G. Caputo, C. Nobile, R. Buonsanti, T. Kipp, L. Manna, R. Cingolani, P.D. Cozzoli, A. Athanassiou, J. Mater. Sci. 43 (2008) 3474.

[16] K. Iketani, R.D. Sun, M. Toki, K. Hirota, O. Yamuguchi, J. Phys. Chem. Solids 64 (2003) 507.

[17] R. Kumar, A.P. Singh, A. Kapoor, K.N. Tripathi, Optik 119 (2008) 553

[18] P.D. Cozzoli, A. Kornowski, H. Weller, J. Am. Chem. Soc. 125 (2003) 14539.

[19] M. Yoshida, P.N. Prasad, Chem. Mater. 8 (1996) 235.

[20] C. Sciancalepore, T. Cassano, M.L. Curri, D. Mecerreyes, A. Valentini, A. Agostiano, R. Tommasi, M. Striccoli, Nanotechnology 19 (2008) 205705.

[21] R.G. Winkler, J.P. Spatz, S. Sheiko, M. Moller, P. Reineker, O. Marti, Phys. Rev. B Cond. Matter 54 (1996) 8908.

[22] S.N. Magonov, V. Elings, M.H. Whangbo, Surf. Sci. 375 (1997) 385.

[23] M. Stark, C. Moeller, D.J. Müller, R. Guckenberg, Biophys. J. 80 (2001) 3009

[24] B. Bushan, J. Qi, Nanotechnology 14 (2003) 886.

[25] A. Mechler, J. Kokavecs, P. Heszler, R. Lai, Appl. Phys. Lett. 82 (2003) 3740.

[26] G. Caputo, C. Nobile, T. Kipp, L. Blasi, V. Grillo, E. Carlino, L. Manna, R. Cingolani, P.D. Cozzoli, A. Athanassiou, J. Phys. Chem. C 112 (2008) 701

[27] A.B.D. Cassie, S. Baxter, Trans. Faraday Soc. 40 (1944) 546

[28] Z. Zhang, O. Bondarchuk, B.D. Kay, J.M. White, Z. Dohnalek, J. Phys. Chem. B 110 (2006) 21840 .

[29] O. Bikondoa, C.L. Pang, R. Ithnin, C.A. Muryn, H. Onishi, G. Thomton, Nat. Mater. 5 (2006) 189. 